\title{
Fabrication and Characterization of low-loss polymeric waveguides and micro-resonators
}

Jacob Scheuer

kobys@eng.tau.ac.il

Amnon Yariv
School of Electrical Engineering, Tel-Aviv University, Ramat-Aviv, Israel

Department of applied physics, California Inst. of Technology, Pasadena, CA

We realize low loss, single-mode polymer waveguides and microring resonators in SU-8 (MichroChem) using direct E-beam lithography. We also present and demonstrate a novel and simple approach for accurately extracting the propagation loss in the waveguides as well as the coupling between the waveguide and the microring. The demonstrated approach is insensitive to the $1 / 0$ coupling efficiency to the optical chip and does not require any pre-calibration of the experimental setup. [DOI: 10.2971/je0s.2006.06007]

Keywords: Waveguides, integrated optics, optical resonators, polymers

\section{Introduction}

The transition from the contemporary low scale integration of optical devices to future highly-integrated photonic processors requires new high-quality materials on one hand and inexpensive mass-production methods on the other. In this context, polymeric materials have interesting optical and mechanical properties, making them an attractive choice for future photonic systems. Compared with other materials, polymeric materials have several inherent advantages:

1. The properties of polymers can be widely tuned by chemically modifying the structure of the monomer, the functional groups or chromophores, or the polymer backbones [1].

2. Their relatively low refractive index results in lower surface-roughness scattering loss and higher coupling efficiency to optical fibers.

3. Polymeric materials can be easily manipulated by several conventional and unconventional fabrication techniques such as dry etching, wet etching, embossing, and soft lithography [1].

4. Compared to fragile glass fiber and expensive semiconductor chips, polymeric materials provide for easy, lowcost, and reliable fabrication of optical devices. In addition, they may serve for rapid prototyping allowing to inexpensive and fast examination of new concepts and devices.

5. Functional polymeric materials also provide an excellent platform for integrating numerous materials with different functions. This ability to dope polymers with diverse molecules, exhibiting large electro-optic coefficient, nonlinear response or optical gain, paves the way to all-polymer integrated optical circuits that include on-chip sources, processors and detectors [2,3].
6. Polymers have also interesting mechanical properties - after curing they are flexible and can, therefore, be bent and attached to non-planar surfaces [4]. In addition, they can be readily integrated with microfluidic structures, shown to be useful for diverse applications such as sensing, imaging, lab-on-a-chip and more [5].

A key ingredient for the realization of polymer-based optical devices and circuitry is the ability to construct and characterize low-loss single-mode waveguides. As a matter of fact, accurate estimation of waveguide propagation loss is a challenging task by itself, especially for low losses.

The most common method to characterize waveguide losses is to measure the transmitted power of a known input after propagating through identical waveguides with different lengths. Alternatively, a single waveguide can be used by sequentially cutting it to shorter lengths and measuring its transmittance (the cutback method). Both configurations suffer from inherent drawbacks - for the first one, it must be assumed that all the measured waveguide are identical and the second one is susceptible to variations of the I/O coupling losses.

Although low-loss polymer waveguides exhibiting propagation losses as low as $0.1 \mathrm{~dB} / \mathrm{cm}$ have been demonstrated before [6]-[12], the waveguides structures described in these studies utilized low index contrast between the core and the cladding $(<1.6 \%)$ and, therefore, unsuitable for compact, densely packed integrated optical components. Here, we demonstrate the realization of low loss polymer waveguides and present a simple though accurate characterization method. The characterization method is based on measuring the spectral properties of micro-ring resonators that are coupled to two straight waveguides (see Figure 1B). The relatively high index contrast 
of the waveguide structure $(5-6 \%)$ allows for the realization of low-loss, small-radius bends that are required for dense integration of optical components. In the second section, we discuss the theoretical background of microring resonators and present the extraction method. In the third section we describe the fabrication of low-loss polymer-based waveguides and microrings, in the forth section we describe the experimental characterization of the microrings and waveguides and in the last section we conclude.

\section{EXTRACTING $M I C R O R$ I N G THE O R Y}

The spectral properties of a coupled waveguide-microring system are completely determined by several parameters: 1) the optical length and dispersion of the waveguide/microring; 2) The waveguide-microring coupling; and 3) The propagation loss in the microring. Therefore, by measuring the spectral properties of such system it is possible to extract these parameters. It should be noted that not every coupled waveguide-microring system is suitable for the extraction of the loss and coupling parameters.

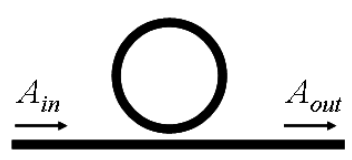

A

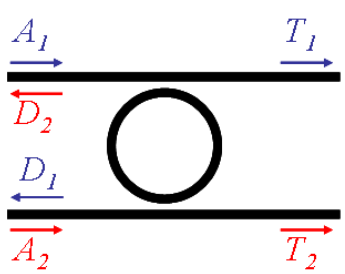

B
FIG. 1 Single (A) and double (B) side coupled ring resonators

For example, in the all-pass configuration (Figure 1A) the transmission spectrum is determined symmetrically by the coupling coefficient and the loss per revolution and thus its measurement cannot distinguish between the two parameters. On the other hand, the double sided coupled waveguide micro-ring system (Figure 1B) does provide enough information to completely characterize the coupling and the loss coefficients and, thus, provides a suitable platform for the characterization of waveguides and microrings.

In order to extract all the parameters of the system it is required to measure the following spectra (see Figure 1B):

1. The reflected signal in port 2 for input in port 1 (or vice versa $)-D_{1}(\omega)$.

2. The transmitted signal in port 3 for input in port $1-$ $T_{1}(\omega)$.

3. The transmitted signal in port 4 for input in port $2-$ $T_{2}(\omega)$.
These spectra are given by [12]:

$$
\begin{aligned}
& D_{1}(\omega)=\frac{-\sqrt{k_{1} k_{2}} \exp (-\alpha L / 4) \exp (i \varphi / 2)}{1-\sqrt{\left(1-k_{1}\right)\left(1-k_{2}\right)} \exp (-\alpha L / 2) \exp (i \varphi)} \\
& T_{1}(\omega)=\frac{\sqrt{\left(1-k_{2}\right)}-\sqrt{\left(1-k_{1}\right)} \exp (-\alpha L / 2) \exp (i \varphi)}{1-\sqrt{\left(1-k_{1}\right)\left(1-k_{2}\right)} \exp (-\alpha L / 2) \exp (i \varphi)} \\
& T_{2}(\omega)=\frac{\sqrt{\left(1-k_{1}\right)}-\sqrt{\left(1-k_{2}\right)} \exp (-\alpha L / 2) \exp (i \varphi)}{1-\sqrt{\left(1-k_{1}\right)\left(1-k_{2}\right)} \exp (-\alpha L / 2) \exp (i \varphi)}
\end{aligned}
$$

where $k_{1}$ and $k_{2}$ are the coupling coefficients at the upper and lower couplers, $\alpha$ is the propagation loss coefficient, $L$ is the ring circumference, $\varphi=\Delta \omega /$ FSR and the FSR (free spectral range) is the spectral separation between successive resonances of the microrong. $\Delta \omega=\omega-\omega_{0}$ is the frequency detuning from the nearest resonance frequency $\left(\omega_{0}\right)$. It should be noted that the spectral response of the Drop port is independent of the input/output port $(1 \rightarrow 2$ or $2 \rightarrow 1)$ while the spectral response of the two Through ports may differ if $k_{1} \neq k_{2}$. This could be due to fabrication errors or even deliberate, especially if it is desired to achieve critical coupling [13].

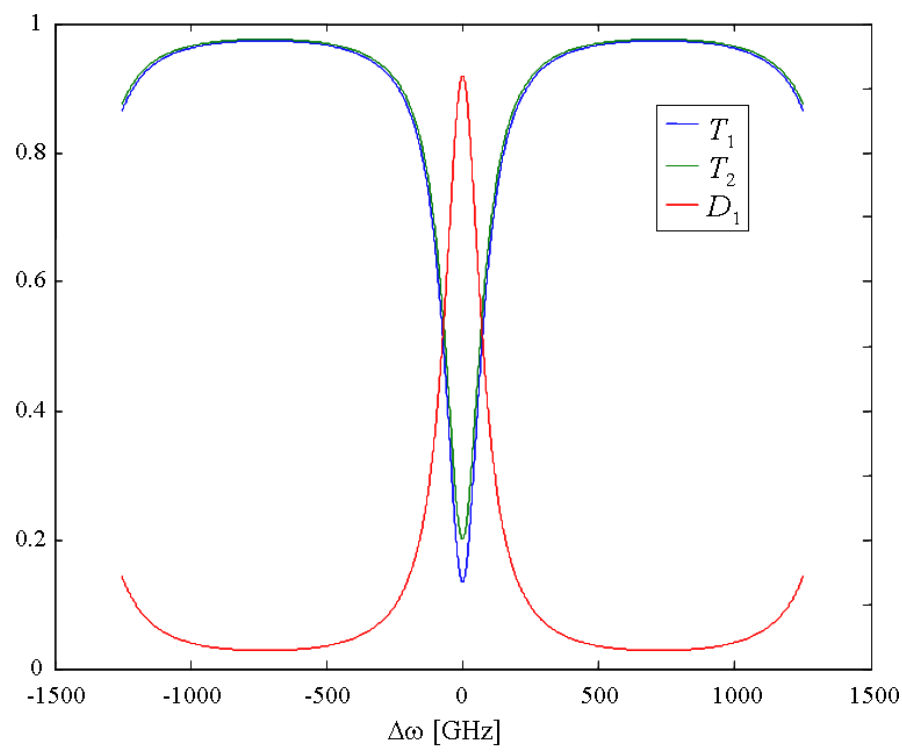

FIC. 2 Theoretically calculated spectral response of the through (blue and green) and drop (red) ports for an asymmetric double side coupled ring resonator.

Figure 2 shows a theoretically calculated $D_{1}, T_{1}$ and $T_{2}$ spectra for $k_{1}=0.2, k_{2}=0.4, \alpha=10 \mathrm{~dB} / \mathrm{cm}$ and $L=125.66 \mu \mathrm{m}$ (corresponding to ring radius of $20 \mu \mathrm{m}$ ). Note that the spectral responses of $T_{1}$ and $T_{2}$ differ because of the different coupling coefficients between the microring and the upper/lower waveguides. One of the important, and relatively difficult to characterize, parameter of any experimental setup involving a microchip is the I/O coupling loss. This loss is determined by the overlap between input/output spots and the mode profile of the characterized waveguide, the quality of the alignment and the facet, etc. The coupling loss affects the overall intensity measured in the output ports and, therefore, without knowing its value it is difficult to compare measurements 
of the signals in different ports. This is because they are not necessarily normalized to the same intensity. Moreover, the I/O coupling loss could also be wavelength dependent, thus adding undesired spectral features to the measured signal.

Thus, in order to provide accurate analysis and parameter evaluation, the extraction of the parameters should not require comparison between signals emerging from different ports (e.g., the through to drop loss) and should be limited to a relatively narrow spectral band. Referring to Eqs.(1a-1c), under the abovementioned restrictions, the following quantities can be simply determined:

\section{The FSR.}

2. Half width half maximum (HWHM) of a resonance drop peak.

3. $T^{\max } / T^{\min }$ for both port 3 and 4 .

Note, that each one these values are extracted from a single port, within a spectral bandwidth of less than a free spectral range. Nevertheless, it is required that the parameters determining the spectral response ( $\mathrm{I} / \mathrm{O}$ coupling loss, $k_{1}, k_{2}$, and $\alpha$ ) remain relatively unchanged within this bandwidth. This, however, is a reasonable requirement which is satisfied in most common material systems and waveguide structures. The abovementioned quantities satisfy the following relations:

$$
\begin{aligned}
& \cos \left(\frac{\Delta \omega_{\text {нғнм }}}{\mathrm{FSR}}\right)=-\frac{1+A^{2}-4 A}{2 A} \\
& \frac{T_{i}^{\max }}{T_{i}^{\min }}=\frac{\left(s_{i}+A\right)(1-A)}{\left(s_{i}-A\right)(1+A)} ; i=1,2
\end{aligned}
$$

where $s_{i}=1-k_{i}$ and $A=\sqrt{s_{1} s_{2}} \exp (1 / 2 \alpha L)$. Eqs.(2a), (2b) can now be used to determine $s_{1,2}$ and $A$ and, correspondingly, the coupling and the propagation loss coefficients:

$$
\begin{gathered}
A=2-\cos \left(\frac{\Delta \omega_{\mathrm{HFHM}}}{\mathrm{FSR}}\right)-\sqrt{\left[2-\cos \left(\frac{\Delta \omega_{\mathrm{HFHM}}}{\mathrm{FSR}}\right)\right]^{2}-1} \\
s_{i}=A\left(1+\frac{(1-A) T_{i}^{\min }}{(1+A) T_{i}^{\max }}\right) /\left(1-\frac{(1-A) T_{i}^{\min }}{(1+A) T_{i}^{\max }}\right) \\
k_{i}=1-s_{i} ; \quad \exp (-\alpha L)=A^{2} / s_{1} s_{2} ; \quad i=1,2
\end{gathered}
$$

Eqs.(3a)-(3c) allow extracting the parameters of the system from relatively simple measurements such as the width of the drop peak and the ratio between the maximal and minimal signal levels at each through port. Note that no numerical fitting is required although such fitting might be useful for obtaining a more accurate estimation of the HWHM.

Few remarks regarding the practical implementation of Eqs.(3a-3c) for waveguide characterization:

1. The propagation loss coefficient, $\alpha$, is actually composed of two contributions - the waveguide propagation loss and the bending losses [14]. Moreover, in most cases the waveguide loss is dominated by the scattering loss which is affected by the intensity of the modal field at the interface between the core and the cladding. At small radii the bending losses become more important and the mode profile deviates significantly from that of a straight waveguide, thus modifying the propagation loss [15]. Therefore, to obtain an accurate estimation of the losses of a straight waveguide it is desired to characterize microrings with increasing radii until the propagation loss becomes independent of the radii. This value is the propagation loss of a straight waveguide.

2. The described method allows also extracting the dependence of the coupling and loss coefficients on the wavelength. Since the determination of the parameters requires only narrow-bandwidth data near a resonance frequency, the procedure can be repeated at each resonance thus sampling the wavelength dependence of the parameters. Larger microrings allow for denser sampling, thus providing more accurate results.

3. The described procedure separates between the I/O coupling loss and the loss in the microchip thus allowing to estimate the overall I/O coupling loss if the input power is know. It also enables comparing between the I/O coupling losses of different ports.

\section{FABRICATION OF POLYMERIC COUPLED WAVEGUIDE-MI- CRORING SYSTEMS}

The waveguides and microrings were fabricated in optical polymeric materials. A silicon wafer with $5 \mu \mathrm{m}$ thermal oxide $(n=1.4455)$ served as the device substrate and lower cladding layer. The core polymer SU-8 $(n=1.565$, available from Microchem Corp.) was cross-linked using direct electron-beam exposure [15]. The waveguides were approximately $2 \mu \mathrm{m}$ in width and $1.8 \mu \mathrm{m}$ in thickness. Finally, in order to reduce the scattering loss, the samples were spin-coated with a UV curable epoxy UV-15 ( $n=1.5$, by Microchem). Finite-difference mode solver calculations showed that the $5 \mu \mathrm{m}$ oxide provides sufficient optical insulation from the $\mathrm{Si}$ substrate and that the waveguides support a single optical mode in the TE polarization.

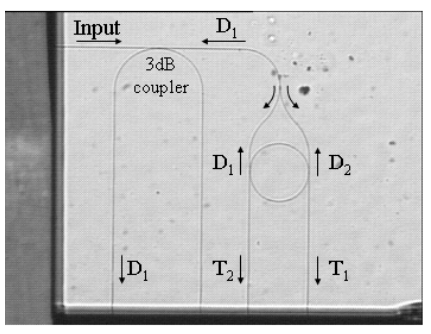

A

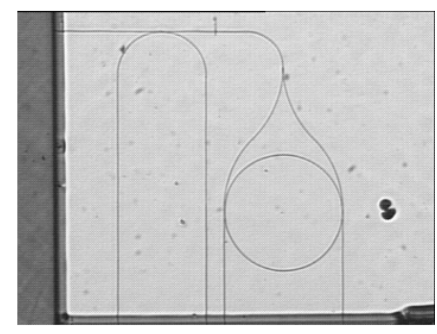

B
FIC. 3 Optical images of the fabricated polymer devices with $\rho=200 \mu \mathrm{m}$ (A) and $\rho=400 \mu m$ (B).

Figure 3 shows an optical images of a typical devices with microring radii of $200 \mu \mathrm{m}$ Eq.(3a), and $400 \mu \mathrm{m}$ Eq.(3b). To sim- 
plify the measurement procedure, a unique configuration, slightly differing than that shown in Figure 1, was adopted. The device operates as follows: light is launched into the input waveguide and is equally divided between the two arms of the $\mathrm{Y}$ junction. The signal in each arm is filtered by the microring, reflecting $D_{1}(\omega)$ and $D_{2}(\omega)$ while transmitting $T_{1}(\omega)$ and $T_{2}(\omega)$. Finally, the two parts of the filtered signal are combined by the $Y$ splitter to generate the Drop signal. The purpose of the $3 \mathrm{~dB}$ coupler (located before the $\mathrm{Y}$ splitter) is to separate between the injected and the reflected signals. Alternatively, one can use a circulator to achieve the same result.

\section{E X P E R I M E N T S}

Figure 4 depicts a schematic of the experimental setup used to characterize the devices. TE polarized light from a tunable laser is coupled into the straight waveguide using a tapered fiber.

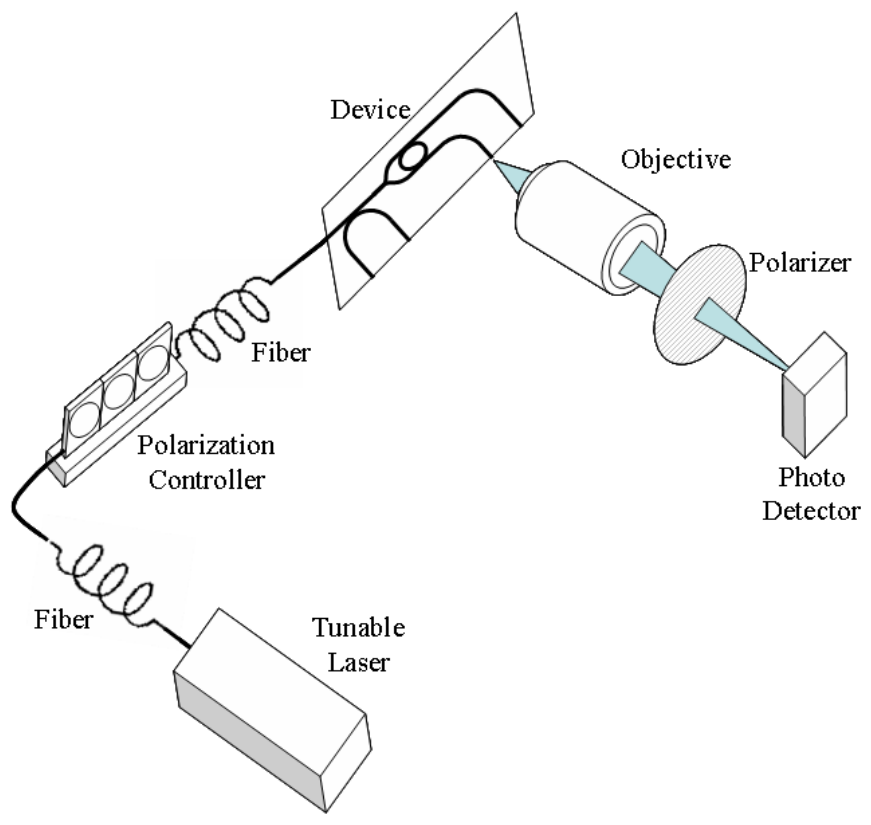

FIG. 4 Schematic of the experimental setup.

The transmission signal, collected by an objective lens from the other end facet of the device, is measured using a femtowatt infrared photoreceiver. The spectral properties of the Drop $\left(D_{1}\right)$ and the two Through $\left(T_{1}, T_{2}\right)$ ports are examined by focusing the light emerging from each port on the detector and scanning the wavelength of the injected light while recording the photoreceiver signal.

Figure 5 depicts a representative spectral response of the three ports for a $300 \mu \mathrm{m}$ radius microring.

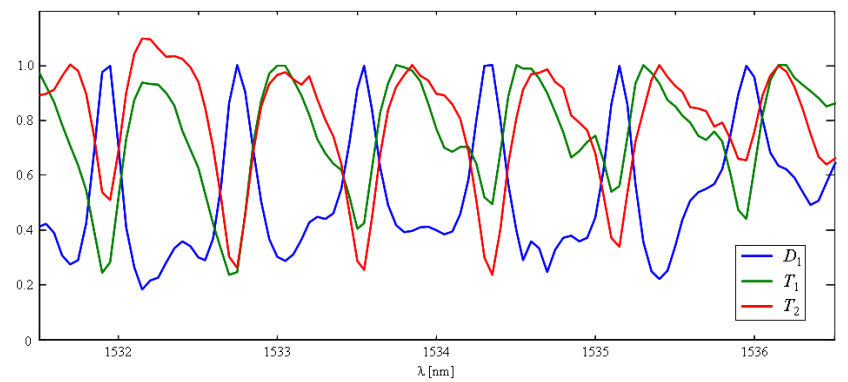

FIG. 5 Measured through and drop transmission function for a $\rho=300 \mu m$ ring resonator.

It should be emphasized that although the microring is not critically coupled (the transmission notches at the through ports are only $-7 \mathrm{~dB}$ deep), the measured data is enough to extract all the parameters of the system. Thus, in contrast to the all-pass configuration, there is no need to tune the waveguidemicroring separation to the critical coupling point. Note, that the depth of the notches at the two through ports $T_{1}$ and $T_{2}$ are not identical, thus indicating a difference between the coupling coefficients $k_{1}$ and $k_{2}$.

To evaluate our waveguides propagation loss, we characterized several microrings of various radii. The resulting loss coefficients are shown in Figure 6: For each radius we plot the extracted propagation loss and coupling coefficients.

A

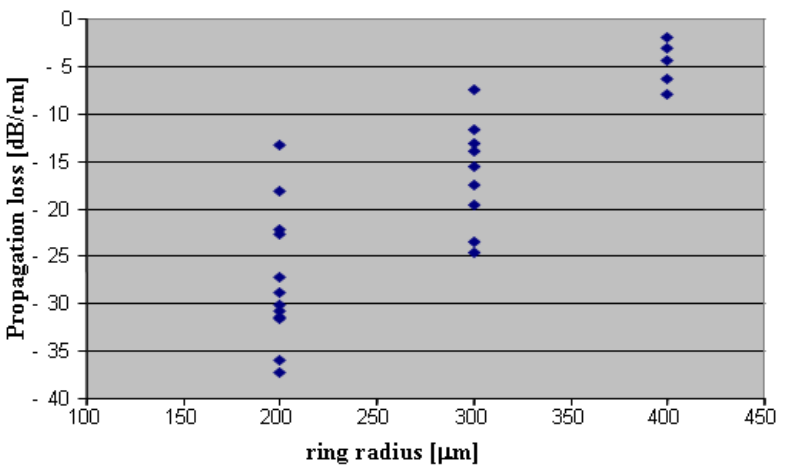

B

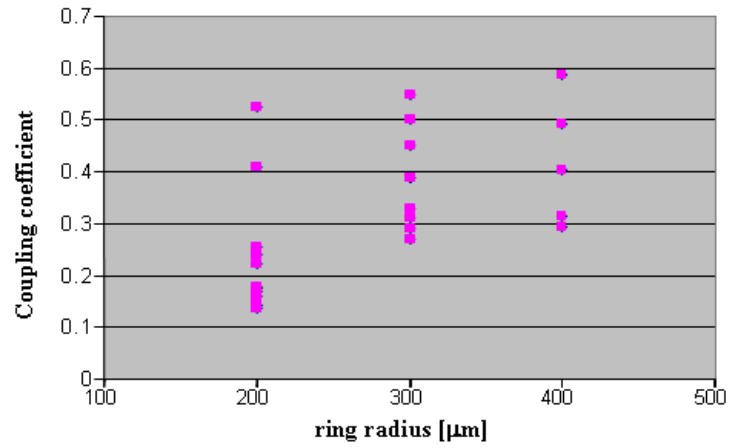

FIC. 6 Propagation loss (A) and coupling (B) coefficients as a function of the microring radius.

The parameters extracted for supposedly identical devices exhibit non-negligible variance which we attribute primarily to inhomogeneous coating of the waveguides core layer across the wafer. Referring to Figure 6A, depicting the propagation loss as a function of the microring radius, it is clear that larger microrings exhibit lower propagation loss. This re- 
sults in not surprising because the bending losses decrease for larger radii. For the larger microring resonators tested, the propagation loss coefficient is as low as $-3 \mathrm{~dB} / \mathrm{cm}$. Note that the expected convergence of the propagation loss to a radius independent value is not apparent in Figure 6, thus indicating that the bending losses are a significant part of the microring losses even at $\rho=400 \mu \mathrm{m}$, indicating that the propagation loss of a straight waveguide is even lower.

\section{C O N CLUSIONS}

We present the realization of low-loss polymer waveguides and microrings using direct E-beam lithography and a novel method for the characterization of their loss and coupling coefficients. The extraction method is inherently normalized and is, therefore, unaffected by the quality of the power coupling into and out of the device. Repeating the measurements for several resonance frequencies of the microrigs allows extracting the wavelength dependence of these coefficients. Waveguides with propagation losses lower than $3 \mathrm{~dB} / \mathrm{cm}$ are demonstrated. By measuring the input and output powers, it is possible to evaluate also the I/O coupling losses as a function of the wavelength. The ability to realize low-loss polymer waveguides and to accurately characterize them is a major milestone in the route to polymer-based optical components and circuitry.

\section{References}

[1] H. Ma, A. K.-Y. Jen and L. Dalton, Advanced Materials 14, 1339 (2002).
[2] J. Scheuer, G. T. Paloczi and A. Yariv, Appl. Phys. Lett. 87, 251102 (2005).

[3] C. Zhang, L. R. Dalton, M.-C. Oh, H. Zahng and W. H. Steir, Chem. Mater. 13, 3043 (2001).

[4] G. T. Paloczi, Y. Huang and A. Yariv, Electron. lett. 39, 1650 (2003).

[5] U. Levy, K. Campbell, A. Groisman, S. Mookherjea and Y. Fainman, Appl. Phys. Lett. 88, 111107 (2006).

[6] B. L. Booth, J. Lightwave Technol. 7, 1445 (1989).

[7] A. Neyer, T. Knoche and L. Muller, Electron. Lett. 29, 399 (1993).

[8] J.-W. Kang, J.-J. Kim, J. Kim, X. Li, and M.-H. Lee, IEEE Photon. Technol. Lett. 14, 1297 (2002).

[9] A. Yeniay, R. Gao, K. Takayama, R. Gao, and A. F. Garito, J. Lightwave Technol. 22, 154 (2004).

[10] N. Yurt, K. Mune, R. Naito, T. Fukuoka, A. Mochizuki, K. Matsumoto, G. Meredith, N. Peyghambarian, and G. E. Jabbour, J. Lightwave Technol. 23, 1291 (2005).

[11] J.-W. Kang, J.-P. Kim, J.-S. Lee, and J.-J. Kim, J. Lightwave Technol. 23, 364 (2005).

[12] C. K. Madsen and J. H. Zhao, Optical Filter Design and Analysis: A Signal Processing Approach (Wiley-Interscience Publications, NewYork, 1999).

[13] A. Yariv, IEEE Photon. Technol. Lett. 14, 483 (2002).

[14] E. A. J. Marcatili, AT \& T Tech. J. 48, 2103 (1969).

[15] D. Rafizadeh, J. P. Zhang, R. C. Tiberio, and S. T. Ho, J. Lightwave Technol. 16, 1308 (1998).

[16] M. Aktary, M. 0. Jensen, K. L. Westra, M. J. Brett, M. R. Freeman, J. Vac. Sci. Technol. B. 21, L5 (2003). 